\title{
Original
}

\section{Zirconia Implant with Rough Surface Produced by YAG Laser Treatment: Evaluation of Histomorphology and Strength of Osseointegration}

\author{
Kae Kakura, Kimie Yasuno, Yusuke Taniguchi, Kazuko Yamamoto, Takuya Sakai, Akihito Irie and Hirofumi Kido
}

Section of Oral Implantology, Department of Oral Rehabilitation, Fukuoka Dental College, Fukuoka, Japan (Accepted for publication, December 10, 2013)

\begin{abstract}
The aims of this study were to produce rough surfaces on zirconia by laser treatment and to examine how changes in surface topography affect tissues surrounding zirconia implants. Threaded zirconia implants with a diameter of $2 \mathrm{~mm}$ and length of $7 \mathrm{~mm}$ were used. The experimental implants had surfaces treated with laser (YAG laser) irradiation (R-ZIs). The controls were not treated with laser irradiation (S-ZrIs). Twenty R-ZIs and twenty S-ZrIs were placed in the tibiae of 8-week-old male SD rats. The peri-implant tissues with implant bodies were collected 4 weeks after implant placement. Light microscopic and histomorphological evaluations were performed, and removal torque (RTQ) was measured. The bone-implant contact (BIC) ratio was approximately 1.25 times higher for R-ZrI than for S-ZrI on the side of the cortical bone, indicating a statistically significant difference $(p<0.05)$. There was no statistically significant difference in their BIC ratios on the side of the bone marrow. On the cortical bone side and bone marrow side, there was no statistically significant difference between $\mathrm{R}-\mathrm{ZrI}$ and $\mathrm{S}-\mathrm{ZrI}$ in the peri-implant bone area (BA), the area of peri-implant bone within the implant threads. RTQ was approximately 7 times higher for R-ZrIs than for S-ZrIs, indicating a statistically significant difference $(p<0.05)$. In this study, the results of the animal experiment revealed new bone formation in the surroundings of the zirconia implants at 4 weeks after implant placement, indicating achievement of osseointegration. The results suggest that laser-produced rough implant surfaces effectively enhance osseointegration.
\end{abstract}

Key words: Yttria-tetragonal zirconia, Surface modification, Osseointegration, YAG laser, Dental implant

\section{Introduction}

Titanium has high processability and is one of the metals with the least amount of ion elution and corrosion compared with other dental metals. Titanium is also highly compatible with bone and is used widely in clinical settings as a dental implant material. The standard of success for titanium implants is the achievement of osseointegration, i.e., a direct contact of bone and implant surface. Previous efforts have been made to enhance osseointegration and to achieve early osseointegration by modifications of implant surface topography ${ }^{1-4)}$. Esthetic problems can arise in metal implants depending on the metal color of the implant material. In addition, metal allergy and hypersensitivity have been reported in a growing number of cases with the increased use of titanium implants ${ }^{5-10)}$. Zirconia ceramics have been reported to be highly useful because of their esthetic properties and biological safety. There are some reports on zirconia ceramics used clinically as implant bodies ${ }^{5-7)}$. They have gained interest as a second generation implant material that has esthetic advantages and has a very low risk for allergy. However, surface modification

Correspondence to: Dr. Kae Kakura, Section of Oral Implantology, Department of Oral Rehabilitation, Fukuoka Dental College, Fukuoka, 814-0193 Japan; Tel: +81-92-801-0411;Fax:+81-92-801-0513;E-mail: okamatsu@college.fdcnet.ac.jp of zirconia ceramics is technically difficult ${ }^{11}$. Thus, only a very small number of studies have been performed on their surface topography and the response of the surrounding tissue ${ }^{8,10,12-19)}$ In the clinical application of zirconia ceramic implant bodies, it is essential to examine the effects of their surface topography on the surrounding tissue. The aims of this study were to produce rough surfaces on zirconia by laser treatment and to examine how changes in surface topography affect tissues surrounding zirconia implants.

\section{Materials and Methods}

\section{Implants}

In this experiment, zirconia ceramic implants were made of yttria-tetragonal zirconia polycrystal (Y-TZP) ceramics. The threaded implants were used with a diameter of $2 \mathrm{~mm}$ and length of $7 \mathrm{~mm}$. Material processing involved zirconia powder (HSY-3FSD: Daiichi Kigenso Kagaku Kogyo Co., Ltd.) subjected to isostatic pressing to form a green body. Green ceramics were machined into a screw shape using diamond tools. They were sintered at $1500^{\circ} \mathrm{C}$, and subsequently the sintered thread areas were subjected to YAG laser irradiation. Laser irradiation parameters were $0.15 \mathrm{~mJ}$ (energy), $3 \mathrm{~W}$ (power), $50 \mathrm{~mm} / \mathrm{s}$ (speed), $5 \mathrm{KHz}$ (Q switch), and $50 \mu \mathrm{m}$ (line width).For the material property of strength, a four-point bending 


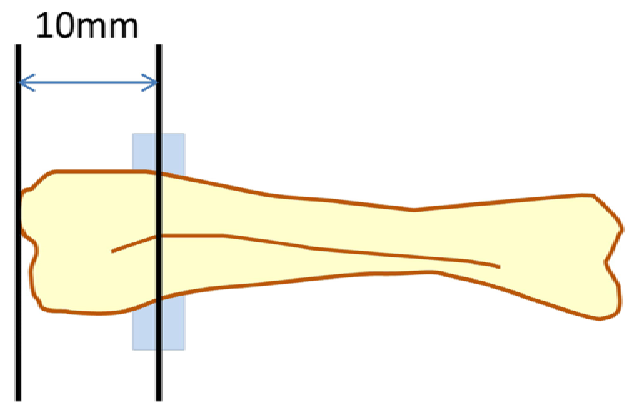

Figure 1. Schematic of implant inserted in a rat tibia. A zirconia implant was placed in a rat tibia at approximately $10 \mathrm{~mm}$ from the knee joint.

test (ISO 14704) was conducted and the mean value was $1167 \pm 225$ $\mathrm{MPa} \pm \mathrm{SD}$. The controls were machined-surfaced zirconia implants (S-ZrI) without laser irradiation. The implants were cleaned ultrasonically in alcohol, dried, and sterilized by autoclave $\left(120^{\circ} \mathrm{C}\right.$ at $2 \mathrm{~atm}$ for $20 \mathrm{~min}$ ).

\section{SEM}

The zirconia implant surfaces were coated with platinum, and their surface topography was examined using a scanning electron microscope (JSM-6330 F, JEOL, Tokyo, Japan).

\section{Topographic evaluation}

The implant surfaces were evaluated according to the guidelines in a previously published repor ${ }^{20}$. Measurements were made at the top of the thread, flank, and valley. Three points were measured in each of these sites, and the mean value of the total of these 9 points was calculated. In our study, the evaluation was performed using three parameters: arithmetic mean height of the surface (Sa), ratio of developed interfacial surface area (Sdr), and density of peaks (Spd) (ISO-25178). The laser microscope used was a Keyence Laser Microscope VK-100 with a filter size of $50 \mu \mathrm{m} \times 50 \mu \mathrm{m}$.

\section{Animal experiment}

Twenty 8-week-old male SD rats were used as experimental animals. Each rat received one experimental implant in one tibia and one control implant in the other tibia (Fig. 1). The rats were given inhalation anesthesia and subsequent intraperitoneal pentobarbital anesthesia $(0.1 \mathrm{mg} / 100 \mathrm{~g})$. The fur surrounding their knee joints was shaved under general anesthesia.

An incision of approximately $15 \mathrm{~mm}$ was made from the knee joint along the anterior border of the tibia. A mucoperiosteal flap was reflected and the bone was exposed. Each implant was placed approximately $10 \mathrm{~mm}$ distal to the tibial knee joint, perpendicular to the tibial long axis, and from the medial to lateral side. After implant placement, the fascia and periosteum were sutured, subsequently the skin was sutured (Coated Vicryl: polyglactin 910), and the surgical treatment was completed. After the surgical treatment, no rat developed complications or died. This study was approved by the Animal Experimentation and Ethics Committee at

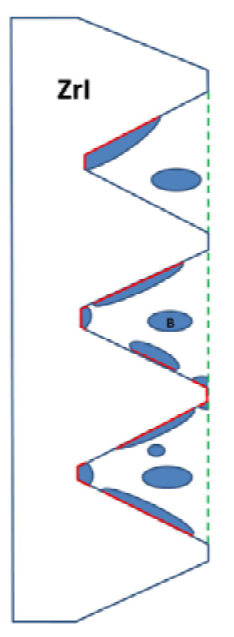

Figure 2. Measurement of tissue surrounding zirconia implant.

ZrI: Zirconia implant. B: Peri-implant bone tissue. BIC: The contact between the bone and implant is shown by a red line. The boneimplant contact ratio (\%) was calculated using the total length of the surface of the three threads used in the measurement. BA: Percentage of bone area in the dotted line (green), connecting the top of the 3 threads that were used in the measurements.

Fukuoka Dental College and performed in accordance with the guidelines on animal experiments.

\section{Histological evaluation}

Four weeks after implant placement, the rats were sacrificed with an overdose of anesthesia. Tibiae, including the implant bodies, were collected as specimens. Immersion fixation was performed on the specimens in $10 \%$ formalin ( $\mathrm{pH} 7.0$ ). Dehydration was performed. The specimens were embedded in MMA resin using a conventional method, and undecalcified ground specimens were prepared and stained with toluidine blue. The undecalcified ground sections were prepared by sectioning the area close to the center of the implant body in the direction of the implant long axis. A light microscope (BX51-DP 12, Olympus, Tokyo, Japan) was used for histomorphological evaluation of the undecalcified ground specimens. For each implant, observation was made on a total of 6 sites in the peri-implant area: 4 sites on the side of the cortical bone and 2 sites on the side of the bone marrow. Measurements were made on 3 threads per site, and evaluation was performed on the bone-implant contact (BIC) ratio and the bone area (BA) within the implant thread (Fig. 2).

\section{Measurement of removal torque}

The tibiae, including implants, were collected from 9 rats at 4 weeks after implant placement to measure the removal torque. The implant bodies were removed under reverse torque rotation using a torque gauge (BTG60CN-S, Tohnichi MFG, Co., Ltd.), and the removal torque was measured.

\section{Statistical analysis}

Statistical analysis was performed using a t-test (two sample assuming equal variance).

\section{Results}

The surface topography of the experimental implant bodies was examined using SEM. The results showed that the experimental 
Kae Kakura et al.: Evaluation of Zirconia Implant with Rough Surface
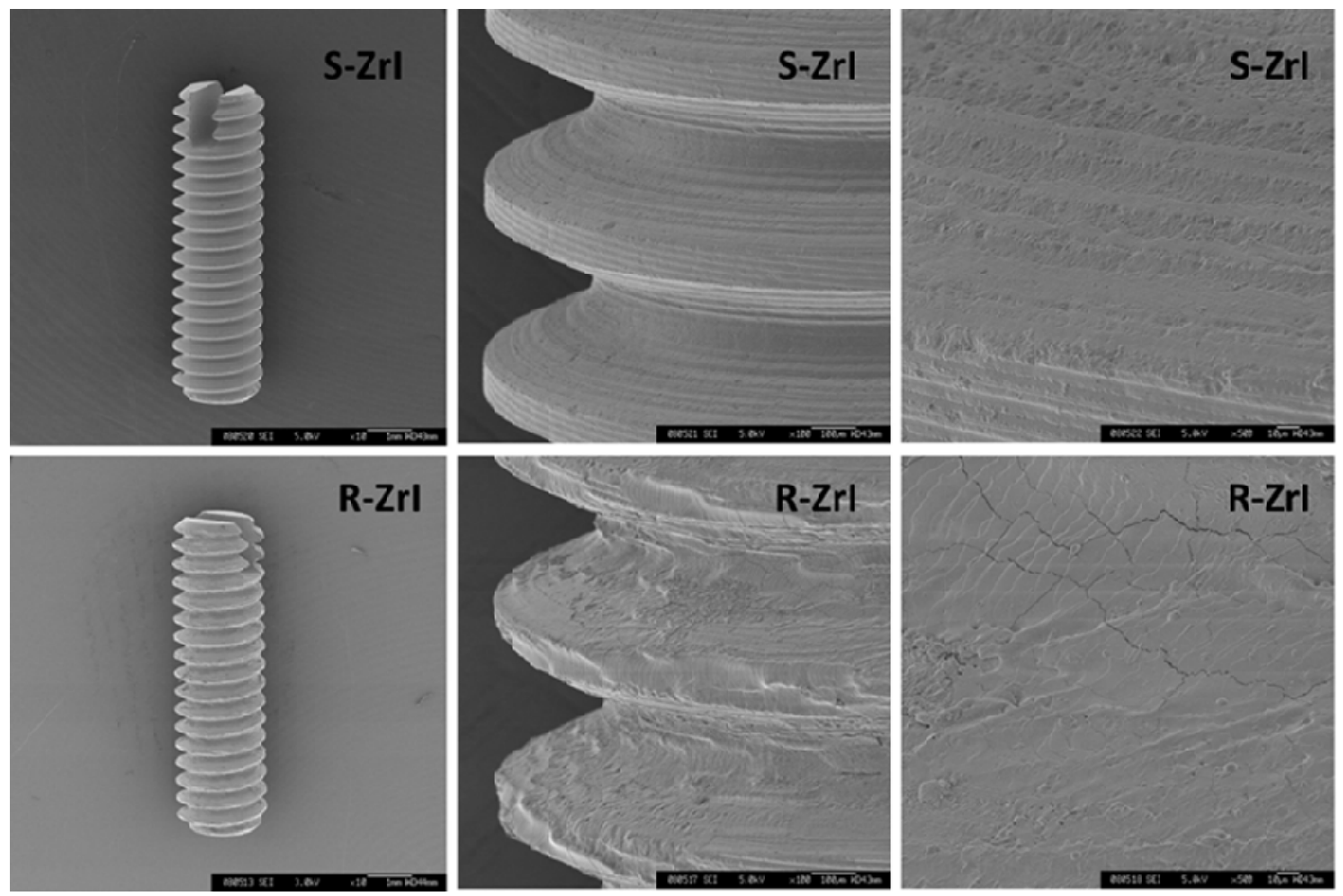

Figure 3. Scanning electron microscopic images of smooth surface zirconia implant (S-ZrI) and rough surface zirconia implant (R-ZrI).
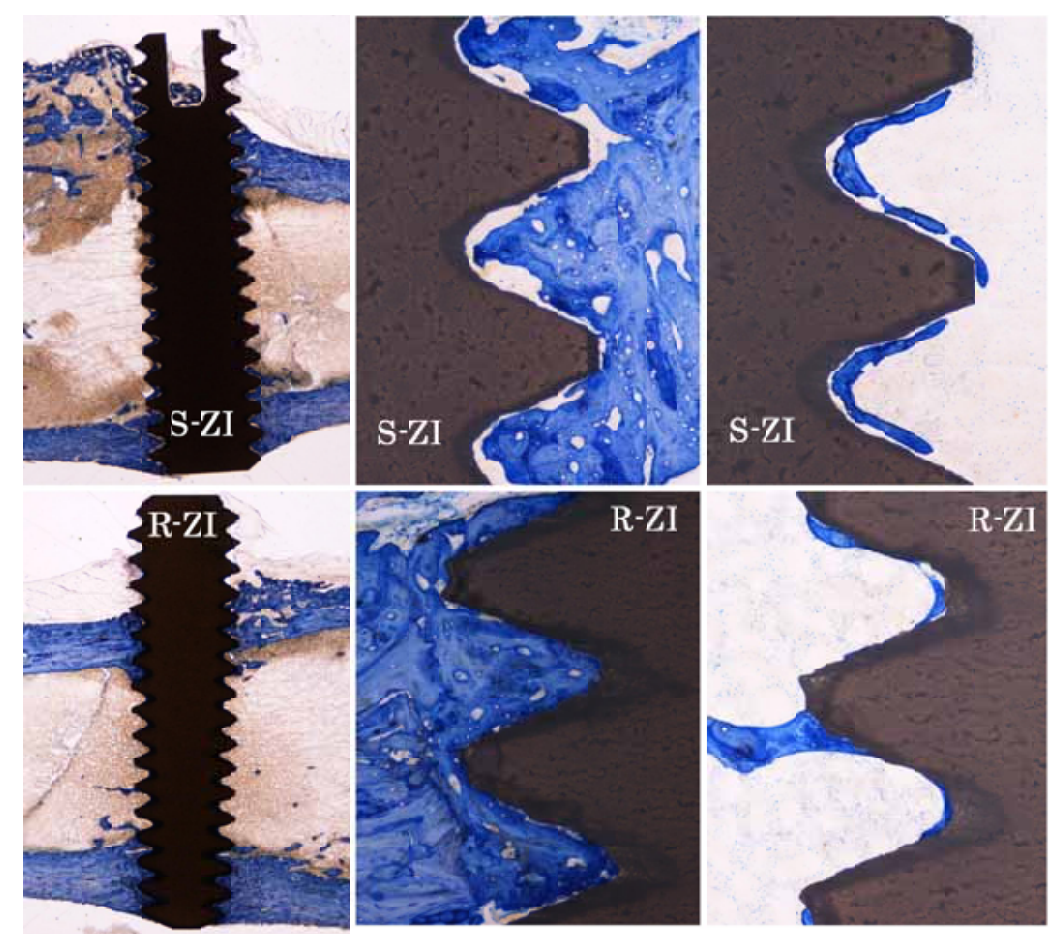

Figure 4. Light micrographs of a smoothsurface zirconia (S-ZrI) implant and a rough-surface zirconia implant (R-ZrI) after healing for 4 weeks. New bone formation was observed in the area surrounding the S-ZrI and along the R-ZrI surface.

implants had characteristic rough surfaces due to laser irradiation compared to the control implant surfaces (Fig. 3). The mean surface roughness of $\mathrm{S}-\mathrm{ZrI}$ and $\mathrm{R}-\mathrm{ZrI}$ was $\mathrm{Sa}=1.06 \pm 0.439 \mu \mathrm{m}$ and $3.33 \pm 2.112 \mu \mathrm{m}$, respectively (Table 1 ). This experiment used a total of 40 implants (20 S-ZrIs and 20 R-ZrIs). No rat showed any findings of inflammation, which could be caused by infection, following wound healing after implant placement surgery. A total of 22 implants were used for undecalcified ground specimens and 18 implants for removal torque measurement. The results of light microscopy revealed that new bone formation occurred around both control and experimental implants at 4 weeks after placement. On the side of the cortical bone, new bone formation was observed 
J.Hard Tissue Biology Vol. 23(1):77-82, 2014

Table 1. Topographic Analyses of the Implants used in This Study

\begin{tabular}{llll}
\hline & $\mathrm{Sa}(\mu 1)$ & $\mathrm{Sdr}(\%)$ & $\mathrm{Spd}\left(\mathrm{mm}^{-2}\right)$ \\
\hline S-ZrI & $1.06 \pm 0.439$ & $11.40 \pm 4.271$ & $530862.44 \pm 283758.860$ \\
$\mathrm{R}-Z r I$ & $3.33 \pm 2.112$ & $49.91 \pm 23.875$ & $588881.18 \pm 163249.211$ \\
\hline
\end{tabular}

S-ZrI: Zirconia implant with machined surface. R-ZrI: Zirconia implant with laser modified surface.

Sa: arithmetic mean height of the surface. Sdr: developed surface area ratio. Spd: peak densities.

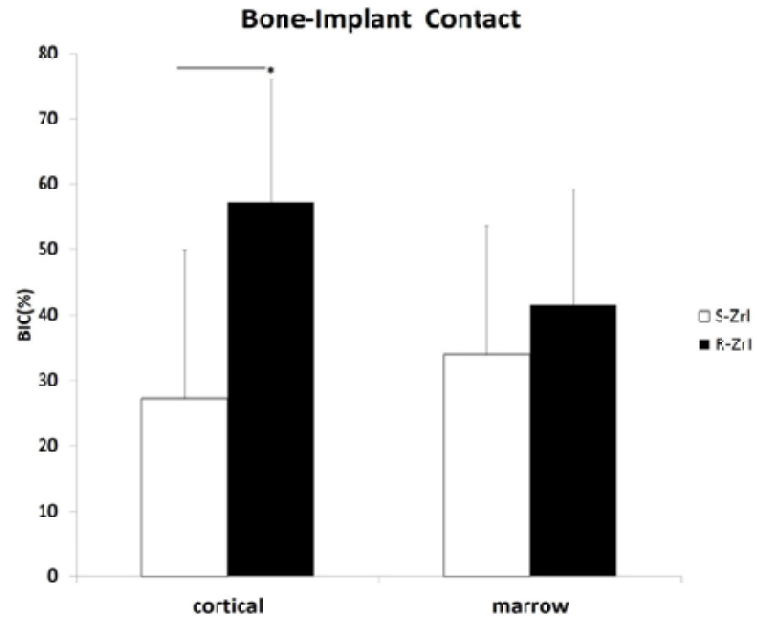

Figure 5. Bone to implant contact (BIC) at 4 weeks after implantation *: statistically significant difference at the cortical bone region $(\mathrm{P}<$ $0.05 ; \mathrm{n}=11)$.

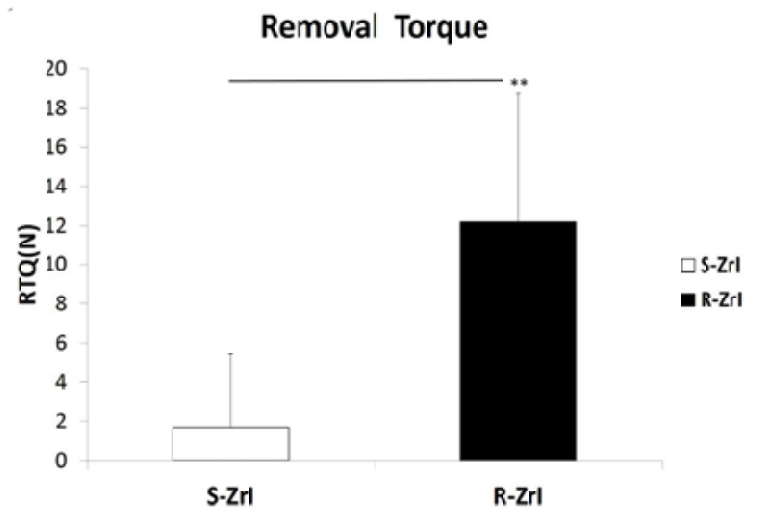

Figure 7. Removal torque (RTQ) at 4 weeks after implantation. **, statistically significant differences $(P<0.05 ; n=9)$.

between the existing bone and implant. On the side of the bone marrow, new bone formation was observed along the implant surface. Thus, achievement of osseointegration was confirmed (Fig. 4). In S-ZrI, the bone-implant contact (BIC) ratio was $27.1 \pm 22.74$ $\%$ on the cortical bone side and $33.92 \pm 19.69 \%$ on the bone marrow side. In R-ZrI, the BIC ratio was $57.26 \pm 18.70 \%$ and $41.50 \pm 17.60$ $\%$, respectively. In S-ZrI, the bone area (BA) was $48.43 \pm 14.17 \%$ and $18.44 \pm 5.10 \%$, respectively. In R-ZrI, the BA was $51.18 \pm 23.74 \%$ and $11.33 \pm 12.56 \%$, respectively. Removal torque was $1.67 \pm 3.74 \mathrm{Ncm}$ for the control implants and $12.19 \pm 6.58 \mathrm{Ncm}$ for the experimental implants (Figs. 5,6,7).

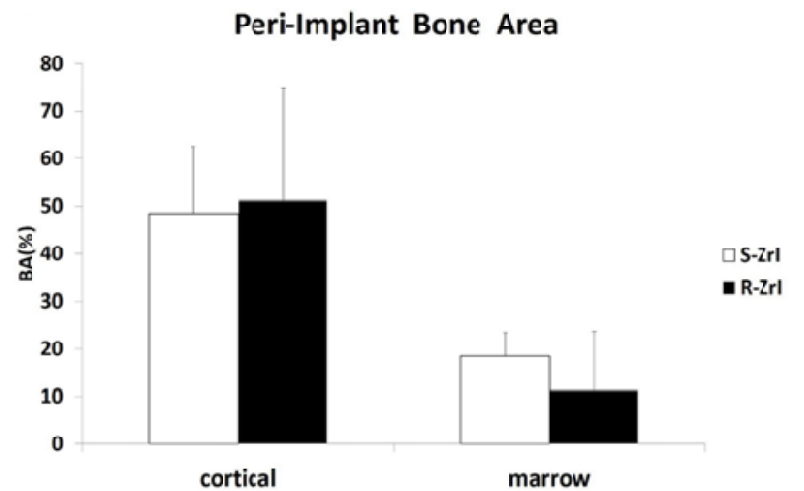

Figure 6. Peri-implant area (BA) at 4 weeks after implantation. There were no statistically significant differences.

\section{Discussion}

In recent years, titanium alloys have become the predominant material used for dental implants. Generally, titanium has high biological safety and a low risk for allergy. However, some patients with titanium implants were reported to have developed symptoms of metal allergy, including facial rash and oral mucosal hyperemia, and underwent removal of implant bodies ${ }^{5}$. Some reports have indicated that titanium allergy and hypersensitivity could cause implant failure ${ }^{7,8)}$. Most commercially available abutments are made of titanium alloy, and other metals in the titanium alloys have been suggested as a possible cause of metal allergy and hypersensitivity ${ }^{10}$. Measures need to be devised against metal allergy and hypersensitivity as the use of titanium implants increases in the future.

Oxide ceramics used in dental materials have biological safety and corrosion resistance superior to metals. In particular, zirconia ceramics have high fracture toughness and satisfactory color tone. Thus, they have been used widely in prostheses and implant abutments in dental clinical settings. Zirconia ceramics are an attractive implant material with high biological safety. However, there are issues with their mechanical properties, including poor flexibility and processability. Therefore, there are far fewer reports on treatment to roughen zirconia ceramic surfaces compared with reports for titanium surfaces. There have been studies regarding surface modifications, involving machining ${ }^{15)}$, sandblasting ${ }^{16-18)}$, and calcium phosphate coating on ceramics ${ }^{14,19)}$. In recent years, several studies have been reported in which zirconia ceramic surfaces were etched or sandblasted much like on titanium implant surfaces ${ }^{21-25}$. Our newly developed zirconia implant (R-ZrI) had surface 


\section{Kae Kakura et al.: Evaluation of Zirconia Implant with Rough Surface}

roughness of $\mathrm{Sa}=3.33 \pm 2.112 \mu \mathrm{m}$. This value is similar to the values of clinically used titanium implants such as plasma-sprayed titanium implants and hydroxyapatite-coated implants. In our study, these rough-surfaced zirconia implants were placed in rat tibiae. The results revealed new bone formation in the peri-implant area at 4 weeks after placement, and osseointegration was achieved.

This study examined how changes in surface topography affect peri-implant tissue. The results showed that the BIC ratio was approximately 1.25 times higher for R-ZrI than for S-ZrI on the cortical bone side $(p<0.05)$. The bone area within the thread was slightly higher for R-ZrI than for S-ZrI on both the cortical bone side and the bone marrow side, but there was no statistically significant difference. RTQ was approximately 7 times higher for $\mathrm{R}-\mathrm{ZrI}$ than for $\mathrm{S}-\mathrm{ZrI}(\mathrm{p}<0.05)$. This finding suggests that removal torque resistance of rough-surfaced implants was positively affected by osseointegration due to new bone formation in the cortical region. The BIC ratio was higher for S-ZrI than for R-ZrI on the bone marrow side. These results suggest that the difference in zirconia surface topography causes the difference in the rate of peri-implant bone formation and the difference in morphology of new bone ${ }^{17,18,24)}$. When the cortical bone side and bone marrow side were examined, there was no difference in BA between S-ZrI and R-ZrI on the cortical bone side. It is thought to be the effect of existing bone. Future studies will be necessary to examine the changes over time in tissue surrounding this newly developed zirconia implant. These results suggests that laser treatment of zirconia implant surface was effective in enhancing osseointegration.

In several past reports, the BIC ratio and BA did not differ greatly between roughsurfaced titanium implants and zirconia implants, or zirconia implants had higher values. However, titanium implants had higher removal torque. Thus, good results have been reported regarding bone formation surrounding zirconia implants. However, the difference in implant materials has been suggested to cause the difference in mechanical strength of osseointegration at the bone-implant interface ${ }^{15,26)}$. There are many studies on the effects of titanium surface roughness. However, studies on zirconia surface topography have just begun. Sennerby et al. ${ }^{19)}$ reported that removal torque increased when osseointegration of the peri-implant area was enhanced due to rough surface processing. However, even if the roughness is similar between titanium and zirconia surfaces, their chemical compositions differ. Thus, it was suggested that the mechanism of osseointegration could be different ${ }^{18)}$. In observations using a SEM, there was no degeneration of surfaces due to YAG laser treatment to produce rough surfaces. However, YAG laser treatment was thought to have damaged portions of the tips of the threads. Stübinger et $\mathrm{al}^{27}$. examined degeneration of zirconia surface caused by irradiation by diode, YAG, and $\mathrm{CO}_{2}$ lasers. Degeneration of surface was reported when a $\mathrm{CO}_{2}$ laser was used.
Future studies need to be performed to examine laser treatment methods that can produce effective rough surfaces. Such examinations include the study of various types of lasers. In addition, examination is needed regarding occlusal load and reaction of intraoral soft tissue for clinical application of our zirconia implant system.

In conclusion, newly developed zirconia implants were subjected to laser treatment to produce rough surfaces and their properties were ivestigated. The implants were placed in rat tibiae. Four weeks after implant placement, new bone formation was observed in the peri-implant areas and osseointegration was achieved. On the cortical bone side, the BIC ratio was approximately 2 times higher and removal torque was approximately 7 times higher for R-ZrI than for S-ZrI, with statistically differences $(p<0.05)$. The results of this study suggest that our zirconia implants were highly biocompatible with periimplant tissue and that surfaces roughened by laser treatment markedly enhanced osseointegration.

\section{Acknowledgements}

The authors are grateful to Nant.Precision CO., LTD. for manufacturing the experimental implants. This work was partly supported by a Strategic Study Base Formation Support Business grant (S 1001059) from the Ministry of Education, Culture, Sports, Science and Technology of Japan.

\section{References}

1. Larsson C, Thomsen P, Aronsson B-O, Rodahl M, Lausmaa J, Kasemo B and Ericson L.E. Bone Response to surfacemodified titanium implants: studies on the early tissue response to machined and electropolished implants with different oxide thicknesses. Biomaterials 17: 605-616, 1996

2. Gottlander M and Albrektsson T. Histomorphometric studies of Hydroxylapatite-coated and uncoated CP titanium threaded implants in bone. Int J Oral Maxillofac Implants 6: 399-404, 1991

3. Buser DL, Schenk R.K, Steinemann S, Fiorellini J.P, Fox C.H and Stich H. Influence of surface characteristics on bone integration of titanium implants. A histomorphometric study in miniature pigs. J Biomed Materi Res 25: 889-902, 1991

4. Buser DL, Broggini N, Wieland M, Schenk RK., Denzer AJ, Cochran DL, Hoffmann B, Lussi A and Steinemann S.G. Enhanced bone apposition to a chemically modified SLA titanium surface. J Dent Res 83: 529-533, 2004

5. Egusa H, Ko N, Shimazu T and Yatani H. Suspected association of an allergic reaction with titanium dental implants: a clinical report. J Prosthet Dent 100: 344-347, 2008

6. Müller K and Valentine-Thon E. Hypersensitivity to titanium: Clinical and laboratory evidence. Neuro Endocrinol Lett 27: 
31-35, 2006

7. Mine Y, Makihira S, Nikawa H, Murata H, Hosokawa R, Hiyama $\mathrm{A}$ and Mimura S. Impact of titanium ions on osteoblast-, osteoclast- and gingival epithelial-like cells. J Prosthodont Res 54:1-6, 2010

8. Thomas P, Bandl W.D, Maier S, Summer B and Przybilla B. Hypersensitivity to titanium osteosynthesis with impaired fracture healing, eczema, and T-cell hyperresponsiveness in vitro: case report and review of the literature. Contact Dermatitis 55: 199-202, 2006

9. Forte G, Petrucci F and Bocca B. Metal allergens of growing significance: epidemiology, immunotoxicology, strategies for testing and prevention. Inflamm Allergy Drug Targets 7: 145162,2008

10. Javed F, Al-Hezaimi K, Almas K and Romanos G.E. Is titanium sensitivity associated with allergic reactions in patients with dental implants? A systematic review. Clin Implant Dent Relat Res 15: 47-52, 2013

11. Subaş1 MG and İnan Ö. Evaluation of the topographical surface changes and roughness of zirconia after different surface treatments. Lasers Med Sci 27:735-742, 2012

12. Oliva J, Oliva $X$ and Oliva JD. Ovoid zirconia implants: anatomic design for premolar replacement. Int J Periodontics Restorative Dent 28: 609-615, 2008

13. Kohal RJ and Klaus G. A zirconia implant-crown system: a case report. Int J Periodontics Restor Dent 24: 147-153, 2004

14. Oliva J, Oliva X and Oliva JD. One-year follow-up of first consecutive 100 zirconia dental implants in humans: a comparison of 2 different rough surfaces. Int J Oral Maxillofac Impl 22: 430-435, 2007

15 Akagawa Y, Ichikawa Y, Nikai H and Tsuru H. Interface histology of unloaded and early loaded partially stabilized zirconia endosseous implant in initial bone healing. J Prosthet Dent 69: 599-604, 1993

16. Kohal R.J, Weng D, Bächle M and Strub J.R. Loaded custommade zirconia and titanium implants show similar osseointegration: an animal experiment. J Periodontol 75: 1262-1268, 2004

17. Gahlert M, Gudehus T, Eichhorn S, Steinhauser E, Kniha H and Erhardt W. Biomechanical and histomorphometric comparison between zirconia implants with varying surface textures and a titanium implant in the maxilla of miniature pigs. Clin Oral Implant Res 18: 662-668, 2007

18. Gahlert M, Röhling S, Wieland M, Sprecher C.M, Kniha H and Milz S. Osseointegration of zirconia and titanium dental implants: a histological and histomorphometrical study in the maxilla of pigs. Clin Oral Implant Res 20: 1247-1253, 2009

19. Sennerby L, Dasmah A, Larsson B and Iverhed M. Bone tissue responses to surfacemodified zirconia implants: A histomorphometric and removal torque study in the rabbit. Clin Implant Dent Relat Res 7: S13-21, 2005

20. Wennerberg A and Albrektsson T. Suggested guidelines for the topographic evaluation of implant surfaces. Int J Oral Maxillofac Implant 15: 331-344, 2000

21. Ferguson SJ, Langhoff JD, Voelter K, von Rechenberg B, Scharnweber D, Bierbaum S, Schnabelrauch M, Kautz AR, Frauchiger VM, Mueller TL, van Lenthe GH and Schlottig F. Biomechanical comparison of different surface modifications for dental implants. Int J Oral Maxillofac Implants 23: 10371046, 2008

22. Langhoff JD, Voelter K, Scharnweber D, Schnabelrauch M, Schlottig F, Hefti T, Kalchofner K, Nuss K and von Rechenberg B. Comparison of chemically and pharmaceutically modified titanium and zirconia implant surfaces in dentistry: a study in sheep. Int J Oral Maxillofac Surg 37: 1125-1132, 2008

23. Depprich R, Zipprich H, Ommerborn M, Naujoks C, Wiesmann H-P, Kiattavorncharoen S. Lauer HC, Meyer U, Kübler NR and Handschel J. Osseointegration of zirconia implants compared with titanium: an in vivo study. Head Face Med. 4:30. doi: 10.1186/1746-160X-4-30, 2008

24. Oliva J, Oliva X and Oliva J.D. Five-year successrate of 831 consecutively placed zirconia dental implants in humans: a comparision of three different rough surfaces. Int J Oral Maxillofac Implant 25: 336-344, 2010

25. Schliephake H, Hefti T, Schlottig F, Gédet P and Staedt H. Mechanical anchorage and peri-implant bone formation of surface-modified zirconia in minipigs. J Clin Periodontol 37: 818-828, 2010

26. Hoffmann O, Angelov N, Zafiropoulos GG and Andreana S. Osseointegration of zirconia implants with different surface characteristics: an evaluation in rabbits. Int J Oral Maxillofac Implants 27: 352-358, 2012

27. Stübinger S, Homann F, Etter C, Miskiewicz M, Wieland M and Sader R. Effect of Er: YAG, $\mathrm{CO}_{2}$ and diode laser irradiation on surface properties of zirconia endosseous dental implants. Lasers Surg Med 40: 223-228, 2008 